\title{
FAUNA DE SCARABAEOIDEA (INSECTA: COLEOPTERA) DE CALMECA, TEPEXCO, PUEBLA, MÉXICO
}

\section{SCARABAEOIDEA BEETLES (INSECTA: COLEOPTERA) OF CALMECA, TEPEXCO, PUEBLA, MEXICO.}

\section{Hortensia CARRILLO-RUIZ, ${ }^{1, *}$ IrMa GUERRA-GONZÁLEZ, ${ }^{1}$ MARCELA SÁNCHEZ-CARRILLO, ${ }^{1}$ Miguel A. MORÓN ${ }^{2}$ y SOMBra P. RIVAS-ARANCIBIA ${ }^{1}$}

\author{
${ }^{1}$ Laboratorio de Entomología, Facultad de Ciencias Biológicas, Benemérita Universidad Autónoma de Puebla, \\ Ciudad Universitaria, Boulevard Valsequillo y Av. San Claudio, Edificio 119A, Col. Jardines de San Manuel, CP \\ 72570 Puebla, México. \\ ${ }^{2}$ Red de Biodiversidad y Sistemática, Instituto de Ecología, A. C. Apartado Postal 63. Xalapa, Veracruz 91000, \\ México <guerra-live@hotmail.com>,<tlaneci.scm@gmail.com>, <miguel.moron@inecol.mx>, \\ <sombrar@gmail.com>. \\ * Autor de correspondencia: <hortensia.carrillo@gmail.com>.
}

Carrillo-Ruiz, H., Guerra-González, I., Sánchez-Carrillo, M., Morón, M. A. y Rivas-Arancibia, S. P. (2017). Fauna de Scarabaeoidea (Insecta: Coleoptera) de Calmeca, Tepexco, Puebla, México. Acta Zoológica Mexicana (n.s.), 33(2), 251-265.

RESUMEN. Se presenta un estudio comparativo de los coleópteros Scarabaeoidea establecidos en la comunidad de Calmeca, Tepexco, Puebla, México. Se realizaron muestreos de noviembre de 2013 a noviembre de 2014 en dos sitios, un sitio de potrero con cultivos aledaños (SP) ubicado a 1,389 msnm y un sitio representativo de selva baja caducifolia con vegetación secundaria (SC) ubicado a 1,441 msnm. Mediante el uso de necrotrampas, trampas de fruta y recolecta directa se obtuvieron 945 ejemplares, representantes de 28 especies distribuidas en seis familias de Scarabaeoidea. La mayor riqueza se presentó en el SP con 27 especies, de las cuales las más abundantes fueron E. intermedius (30.19\%) con 257 ejemplares, seguida de D. gazella (14.33\%) con 122 ejemplares. Por otra parte en el SC las especies más abundantes fueron C. (C.) humectus (20.21\%) y C. (C.) pluto (20.21\%) cada una con 19 ejemplares recolectados. Con respecto a la diversidad, no se encontraron diferencias significativas entre los dos sitios de muestreo $(p=0.111)$. La comunidad de escarabajos de Calmeca se agrupó en ocho gremios alimentarios. En ambos sitios la mayoría de las especies registradas pertenecen a los gremios coprófago (SP 37\% y SC 53\%), necrófago (SP 14.8\% y SC 26.6\%) y filófago (SP 18\% y SC $13 \%)$. El SP presentó una mayor riqueza funcional (ocho gremios) que el SC (cuatro gremios), en este último no se registraron especies de los gremios depredador, antófilo, rizófago y detritívoro.

Palabras Clave: Scarabaeoidea, riqueza, abundancia, gremios alimentarios.
Carrillo-Ruiz, H., Guerra-González, I., Sánchez-Carrillo, M., Morón, M. A., \& Rivas-Arancibia, S. P. (2017). Scarabaeoidea beetles (Insecta: Coleoptera) of Calmeca, Tepexco, Puebla, Mexico. Acta Zoológica Mexicana (n.s.), 33(2), 251-265.

ABSTRACT. A comparative study of Coleoptera Scarabaeoidea in the community of Calmeca, Tepexco, Puebla, Mexico is presented. Samples of beetles were taken from November 2013 to November 2014 in a place with induced pasture land with neighboring crops (SP) and a site representative of deciduous forest with secondary vegetation (SC). By mean of necrotraps, rotten fruit traps and hand collecting, 945 specimens were obtained, representing 28 species in six families of Scarabaeoidea. The SP had the highest species richness with 27 species; the most abundant species was E. intermedius (30.19\%) with 257 specimens, followed by D. gazella (14.33\%) with 122 specimens. In the SC the most abundant species were C. (C.) humectus (20.21\%) and C. (C.) pluto (20.21\%) each with 19 specimens collected. No significant differences between the two sampling sites $(p=0.111)$ were found. The Calmeca beetle community was grouped in eight feeding guilds. At both sites most recorded species belong to the coprophagous guild (SP 37\% \& SC 53\%), the necrophagous guild (SP 14.8\% \& SC $26.6 \%$ ) and the phytophagous guild (SP 18\% \& SC 13\%). The SP had a higher functional richness with eight guilds, compared with the SC with four guilds; in the SC no species of predator, antophilous, and rhizophagus guilds were recorded.

Key words: Scarabaeoidea, richness, abundancy, alimentary guilds. 


\section{INTRODUCCIÓN}

Uno de los principales retos en los diferentes grupos biológicos es la obtención de información precisa sobre la distribución de especies (Castañeda-Osorio et al., 2015; Trujillo-Miranda et al., 2016). En México, se han citado cerca de 1,700 de las 30,000 especies de Scarabaeoidea catalogadas en el mundo. A la fecha se ha reunido información que ha permitido conocer la distribución y hábitos de unas 900 especies de estos coleópteros, faltando datos para unas 800 especies (Morón, 2013a). Además, existen localidades en el país donde se desconocen las especies que en ellas habitan. Morón (2013a) calcula que faltan aproximadamente 400 especies de Scarabaeoidea por registrarse en México.

La riqueza de especies es diferente para cada entidad federativa, ya que depende de la variedad de ambientes representados en cada región geográfica y de la filiación de los taxa, sin embargo, los registros disponibles están influidos por la intensidad con la que se ha explorado la fauna de escarabajos de cada localidad (Morón, 2003). En el estado de Puebla, entre 1996 y 2006, se han efectuado recolectas sistemáticas y esporádicas de coleópteros escarabaeoideos; de los 217 municipios que integran la entidad, se han realizado estudios sistemáticos de los escarabajos en 20 municipios, y en 48 municipios se han recolectado de manera esporádica, entre estos últimos se encuentra el municipio de Tepexco (Jiménez-García et al., 2013) (Fig. 1), ya que hasta el momento se cuenta con el estudio de los escarabajos necrófilos de un sitio conservado de bosque tropical deciduo, en la localidad de Tepexco, en los límites del municipio de Tepexco, (Puebla) con el estado de Morelos, capturados con trampa NTP-80, de febrero a noviembre de 1992 (Deloya, 1992).

Para contribuir al conocimiento de la diversidad de este grupo, se planteó este trabajo en la comunidad de Calmeca, del municipio de Tepexco, Puebla, y comparar la riqueza, abundancia y diversidad de la comunidad de especies de Scarabaeoidea en dos ambientes, natural conservado e inducido, así como proporcionar información sobre el esfuerzo de muestreo y la diversidad funcional.

\section{MATERIALES Y MÉTODOS}

Descripción del área de estudio. El municipio de Tepexco se encuentra al suroeste del estado de Puebla, entre 1,100 y 1,700 msnm (INAFED-SEGOB, 2010); la localidad urbana de Calmeca, se encuentra ubicada a
18 37“59.9”N y 98 37’59.9”' O (Fig. 2), el clima que predomina es cálido subhúmedo (Awo) con lluvias en verano y con una precipitación pluvial de 800 a $1000 \mathrm{~mm}$ anuales. La temperatura media anual oscila entre $21^{\circ} \mathrm{y}$ $25^{\circ} \mathrm{C}$ (INEGI, 2009; Jiménez-García et al., 2013). La vegetación predominante es selva baja caducifolia, la cual ha sido modificada para introducir cultivos y potreros (Jiménez-García et al., 2013), quedando algunos remanentes de este tipo de vegetación en sus alrededores. Para realizar este estudio se seleccionaron dos sitios, fuera de la localidad urbana: sitio de potrero con cultivos aledaños (SP) ubicado a $1,389 \mathrm{msnm}$ y $18^{\circ} 40^{\prime} 00.8^{\prime \prime} \mathrm{N}$ y $98^{\circ} 37^{\prime}$ 27.1" W; y un sitio conservado (SC), representativo de selva baja caducifolia con vegetación secundaria (estratos arbóreos y arbustivos) ubicado a 1,441 msnm y 18 40' 33.7” N y 98 37' 37.1” W.

Métodos de Muestreo. En cada uno de los sitios, se efectuaron muestreos diurnos sistemáticos mensuales de adultos escarabaeoideos, en un periodo de noviembre de 2013 a noviembre de 2014, durante tres días cada mes. En cada sitio de muestreo se trazó un transecto de $100 \mathrm{~m}$, en el cual se instalaron dos tipos de trampas intercaladas y separadas por 10 metros entre sí, cinco necrotrampas NTP 80 (Morón \& Terrón, 1988) cebadas con calamar en descomposición y cinco trampas de fruta, cebadas con fermentado de plátano, cerveza, vainilla y azúcar, estas últimas colocadas en la copa de los árboles a una altura superior a los dos metros (Rivera-Gasperín et al., 2013). Además, en cada sitio se realizó recolecta directa en sitios aledaños al transecto, revisando el follaje de las plantas, la hojarasca, troncos podridos, en las flores, bajo piedras y en excremento, con un esfuerzo de 9h/hombre, en un horario de 8:00 a 17:00 hrs. No se realizaron recolectas nocturnas. Los organismos obtenidos fueron procesados para su montaje en seco e identificados con la clave de las especies de Coleoptera Scarabaeoidea del estado de Puebla (Morón, 2013b). Los ejemplares se encuentran depositados en la colección entomológica del Instituto de Ecología A. C. (IEXA).

Métodos para el análisis de datos. Para evaluar la eficiencia de muestreo, se obtuvo el índice de complementariedad de los dos sitios de la zona de estudio (Villarreal et al., 2004), y posteriormente se obtuvo la curva de acumulación de especies (Jiménez-Valverde \& Hortal, 2003). Para el análisis se emplearon dos estimadores no paramétricos: Chao1 y ACE, ya que estos no se ajustan a un modelo matemático determinado y proporcionan información de los datos con base en las abundancias de las especies registradas. Los estimadores se obtuvieron con 


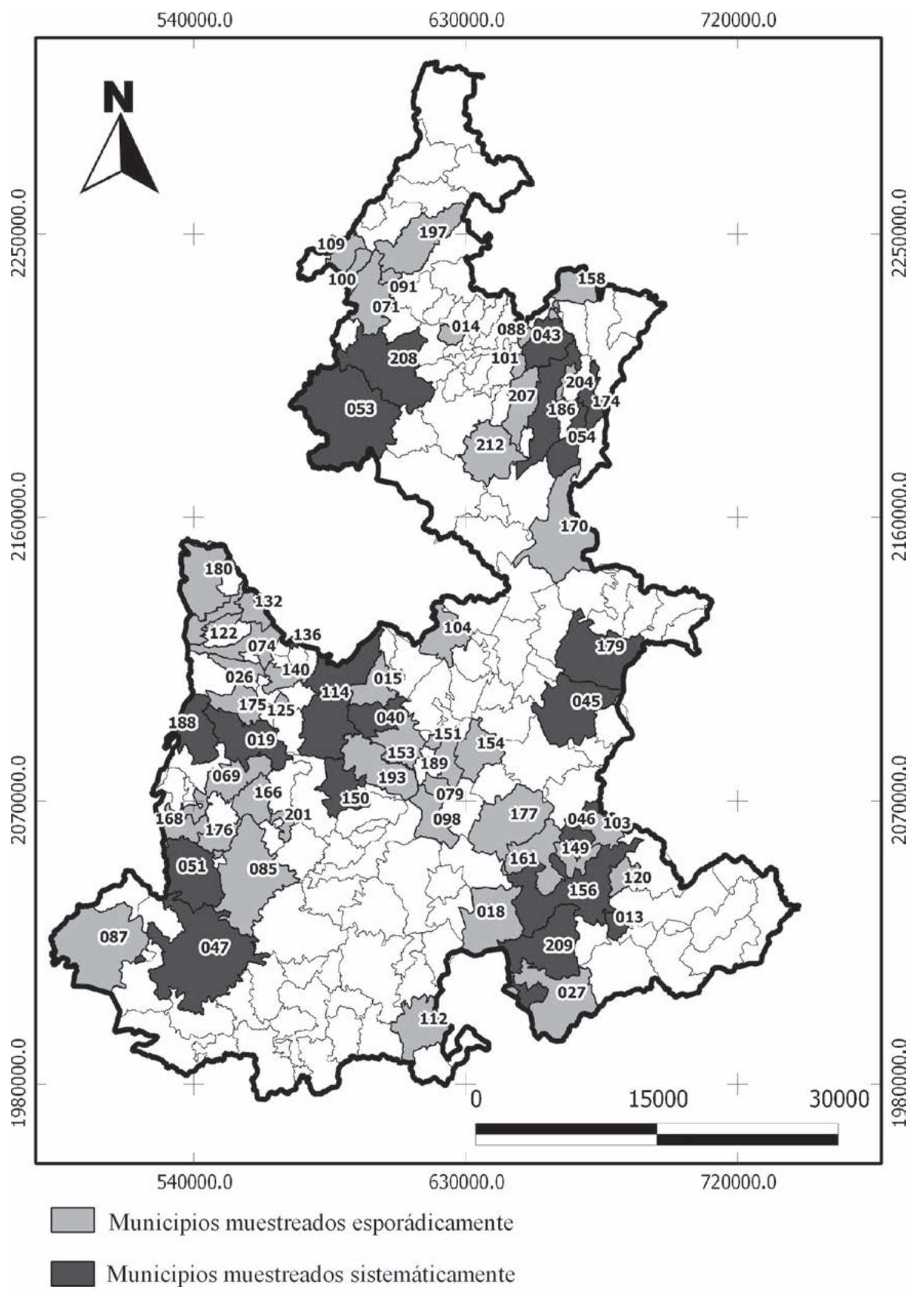

Figura 1. Municipios del estado de Puebla, con estudios sistemáticos a mediano plazo y municipios con estudios esporádicos, entre los que se encuentra Tepexco (168) (Jiménez-García et al., 2013). Los números corresponden a las claves oficiales de los municipios. 


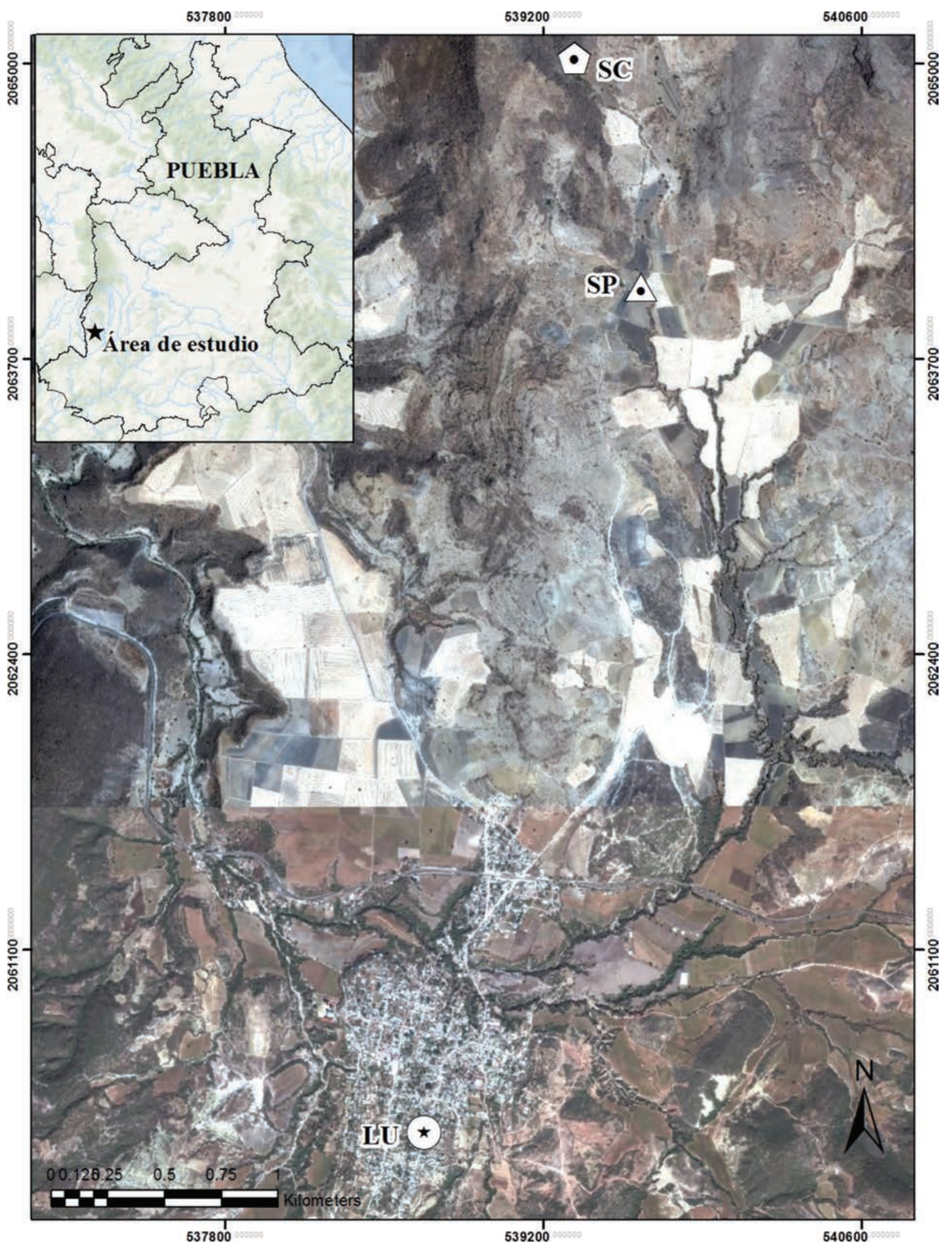

Figura 2. Área de estudio en Calmeca, Tepexco, Puebla, México (LU= zona urbana de Calmeca; $\mathrm{SC}=$ sitio conservado; $\mathrm{SP}=$ sitio modificado). 
el programa EstimateS V.9.1.0 (Colwell, 2013). Se obtuvo la riqueza específica y la abundancia relativa de cada uno de los sitios muestreados (SP y SC) y se realizaron diagramas de rango-abundancia de las especies de escarabaeoideos por sitio (Magurran, 2004). Se calculó el índice de diversidad de Shannon para cada sitio de trabajo y se compararon por medio de un análisis de T de Hutcheson (Hutcheson 1970), para lo cual se empleó el programa estadístico PAST ver $2.17 \mathrm{c}$ (Hammer et al., 2001).

Para determinar la diversidad funcional se realizó la búsqueda de información bibliográfica y se determinó el gremio alimentario de las especies de escarabaeoideos de la zona de estudio. Se generalizó el gremio del género para aquellas especies de las cuales no se encontró información específica. La diversidad funcional se midió como la frecuencia de los gremios alimentarios en los sitios de estudio (García-Martínez et al., 2015) y para comparar la diversidad funcional entre los sitios de estudio se empleó el índice de similitud funcional de Sörensen, modificado por Silvestre (2000), el cual incluye el número de grupos funcionales (gremios alimenticios) en cada sitio y el número de especies dentro de cada gremio.

\section{RESULTADOS}

En los dos sitios de estudio, se registraron 945 ejemplares, de 28 especies distribuidas en seis familias: Melolonthi- dae, Cetoniidae, Scarabaeidae, Hybosoridae, Trogidae y Passalidae. A continuación se muestra la composición específica para cada género.

\section{Melolonthidae, Melolonthinae, Diplotaxini}

Diplotaxis Kirby. D. consentanea Bates, 1887; cuatro ejemplares en abril y mayo (2014), tres ejemplares en SP y uno en SC, con trampa de fruta (1), necrotrampa (1) y recolecta directa (2) (Cuadros 1-2). En Puebla esta especie se ha registrado en Jolalpan, Teziutlán y el Valle de Puebla (Cuadro 3).

\section{Melolonthidae, Melolonthinae, Melolonthini}

Phyllophaga Harris. P. (Phyllophaga) fulviventris Moser, 1918, un ejemplar (junio 2014), capturado con trampa de fruta en SC (Cuadro 1); en el estado de Puebla se ha registrado en Chiautla de Tapia, Huehuetlán el Grande y Jolalpan (Cuadro 2); P.(Phyllophaga) ravida Blanchard, 1850, dos ejemplares (mayo 2014), en hojarasca en SP (Cuadros 1-2). En Puebla se ha registrado en Chiautla de Tapia, Cuetzalan, Huehuetlán el Grande, PopocatépetlTochimilco, el Valle de Puebla y el Valle de Tehuacán (Cuadro 3); P. (Phyllophaga) setifera Burmeister,1855, un ejemplar (mayo 2014) sobre vegetación en SP (Cua-

Cuadro 1. Se muestran las especies colectadas y sus abundancias en los sitios estudiados en Calmeca, Tepexco, Puebla, México. (Código= Número correspondiente a la curva de acumulación; SP= sitio modificado; SC= sitio conservado).

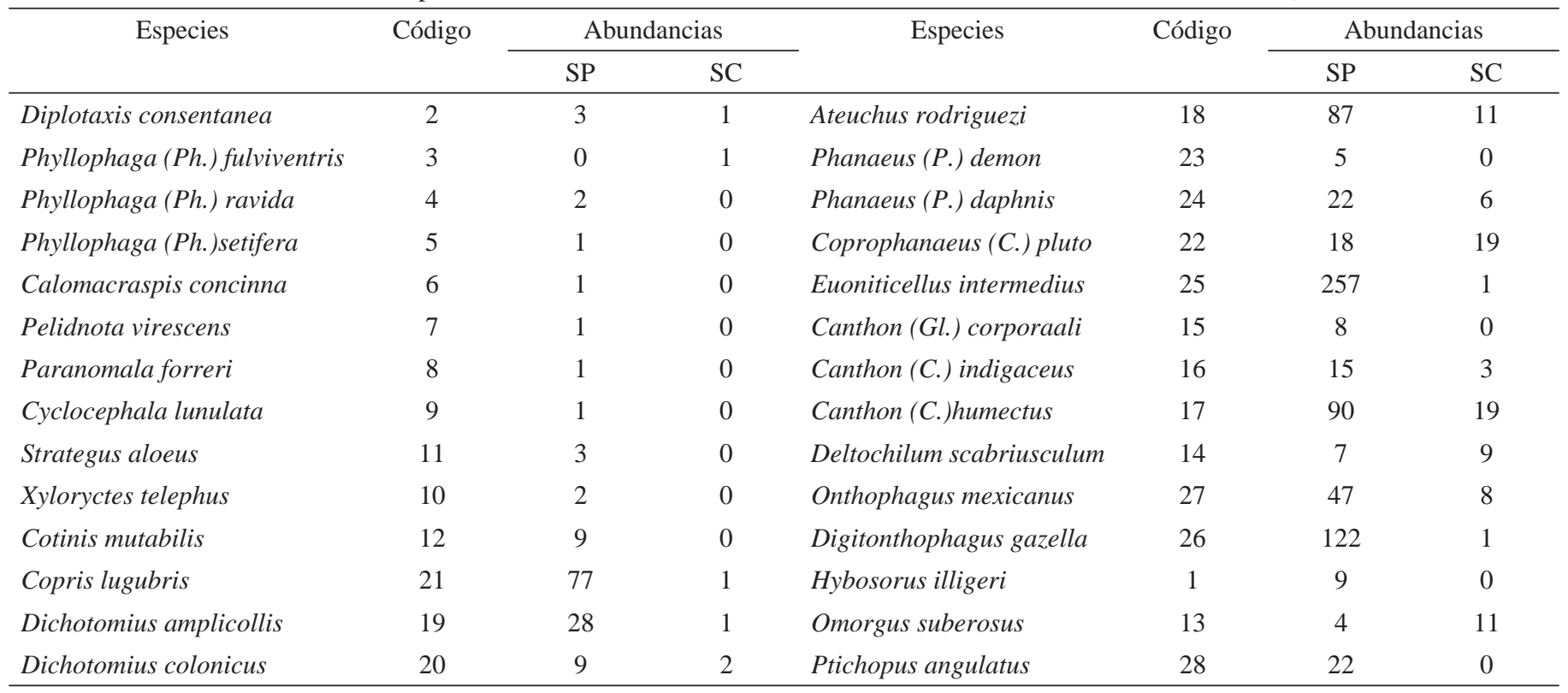


Cuadro 2. Relación de captura de especies en los sitios estudiados en Calmeca, Tepexco, Puebla, México, con los tres tipos de muestreo: colecta directa, necrotrampa NTP-80 (NTP) y trampa de fruta (TF) $(\mathrm{H}=$ hojarasca; $\mathrm{SV}=$ sobre vegetación; $\mathrm{S}=$ suelo; $\mathrm{BGV}=$ boñiga de ganado vacuno; $\mathrm{TD}=$ troncos derribados).

\begin{tabular}{|c|c|c|c|c|c|c|c|c|c|c|c|c|c|c|}
\hline \multirow[t]{3}{*}{ Especie } & \multicolumn{7}{|c|}{ Sitio de potrero } & \multicolumn{7}{|c|}{ Sitio conservado } \\
\hline & \multicolumn{5}{|c|}{ Colecta directa } & \multirow[t]{2}{*}{ NTP } & \multirow[t]{2}{*}{$\mathrm{TF}$} & \multicolumn{5}{|c|}{ Colecta directa } & \multirow[t]{2}{*}{ NTP } & \multirow[t]{2}{*}{ TF } \\
\hline & $\mathrm{H}$ & SV & $\mathrm{S}$ & BGV & TD & & & $\mathrm{H}$ & SV & $\mathrm{S}$ & BGV & TD & & \\
\hline D. consentanea & 0 & 2 & 0 & 0 & 0 & 1 & 0 & 0 & 0 & 0 & 0 & 0 & 0 & 1 \\
\hline Ph. (Ph.) fulviventris & 0 & 0 & 0 & 0 & 0 & 0 & 0 & 0 & 0 & 0 & 0 & 0 & 0 & 1 \\
\hline Ph. (Ph.) ravida & 2 & 0 & 0 & 0 & 0 & 0 & 0 & 0 & 0 & 0 & 0 & 0 & 0 & 0 \\
\hline Ph. (Ph.) setifera & 0 & 1 & 0 & 0 & 0 & 0 & 0 & 0 & 0 & 0 & 0 & 0 & 0 & 0 \\
\hline C. concinna & 0 & 1 & 0 & 0 & 0 & 0 & 0 & 0 & 0 & 0 & 0 & 0 & 0 & 0 \\
\hline P. virescens & 0 & 1 & 0 & 0 & 0 & 0 & 0 & 0 & 0 & 0 & 0 & 0 & 0 & 0 \\
\hline P. forreri & 0 & 1 & 0 & 0 & 0 & 0 & 0 & 0 & 0 & 0 & 0 & 0 & 0 & 0 \\
\hline C. lunulata & 0 & 0 & 1 & 0 & 0 & 0 & 0 & 0 & 0 & 0 & 0 & 0 & 0 & 0 \\
\hline S. aloeus & 0 & 0 & 3 & 0 & 0 & 0 & 0 & 0 & 0 & 0 & 0 & 0 & 0 & 0 \\
\hline X. telephus & 0 & 0 & 2 & 0 & 0 & 0 & 0 & 0 & 0 & 0 & 0 & 0 & 0 & 0 \\
\hline C. mutabilis & 0 & 9 & 0 & 0 & 0 & 0 & 0 & 0 & 0 & 0 & 0 & 0 & 0 & 0 \\
\hline C. lugubris & 0 & 0 & 0 & 77 & 0 & 0 & 0 & 0 & 0 & 0 & 1 & 0 & 0 & 0 \\
\hline D. amplicollis & 0 & 0 & 0 & 17 & 0 & 11 & 0 & 0 & 0 & 0 & 0 & 0 & 1 & 0 \\
\hline D. colonicus & 0 & 0 & 0 & 9 & 0 & 0 & 0 & 0 & 0 & 0 & 2 & 0 & 0 & 0 \\
\hline A. rodriguezi & 0 & 0 & 0 & 72 & 0 & 15 & 0 & 0 & 0 & 0 & 2 & 0 & 9 & 0 \\
\hline P. (P.) demon & 0 & 0 & 0 & 5 & 0 & 0 & 0 & 0 & 0 & 0 & 0 & 0 & 0 & 0 \\
\hline P. (P.) daphnis & 0 & 0 & 0 & 17 & 0 & 5 & 0 & 0 & 0 & 0 & 0 & 0 & 6 & 0 \\
\hline C. (C.) pluto & 0 & 0 & 0 & 0 & 0 & 18 & 0 & 0 & 0 & 0 & 0 & 0 & 19 & 0 \\
\hline E. intermedius & 0 & 0 & 0 & 257 & 0 & 0 & 0 & 0 & 0 & 0 & 1 & 0 & 0 & 0 \\
\hline C. (Gl.) corporaali & 0 & 0 & 0 & 8 & 0 & 0 & 0 & 0 & 0 & 0 & 0 & 0 & 0 & 0 \\
\hline C. (C.) indigaceus & 0 & 0 & 0 & 7 & 0 & 8 & 0 & 0 & 0 & 0 & 0 & 0 & 3 & 0 \\
\hline C. (C.)humectus & 0 & 0 & 0 & 81 & 0 & 9 & 0 & 0 & 0 & 0 & 3 & 0 & 16 & 0 \\
\hline D. scabriusculum & 0 & 0 & 0 & 0 & 0 & 7 & 0 & 0 & 0 & 0 & 0 & 0 & 9 & 0 \\
\hline O. mexicanus & 0 & 0 & 0 & 47 & 0 & 0 & 0 & 0 & 0 & 0 & 5 & 0 & 3 & 0 \\
\hline D. gazella & 0 & 0 & 0 & 122 & 0 & 0 & 0 & 0 & 0 & 0 & 1 & 0 & 0 & 0 \\
\hline H. illigeri & 0 & 0 & 3 & 6 & 0 & 0 & 0 & 0 & 0 & 0 & 0 & 0 & 0 & 0 \\
\hline O. suberosus & 0 & 0 & 0 & 0 & 0 & 4 & 0 & 0 & 0 & 0 & 0 & 0 & 11 & 0 \\
\hline P. angulatus & 0 & 0 & 0 & 0 & 22 & 0 & 0 & 0 & 0 & 0 & 0 & 0 & 0 & 0 \\
\hline
\end{tabular}

dros 1-2); en Puebla se ha registrado en Huehuetlán el Grande y el Valle de Puebla (Cuadro 3).

\section{Melolonthidae, Rutelinae, Rutelini}

Calomacraspis Bates. C. concinna Blanchard, 1850, un ejemplar (junio 2014) sobre vegetación en SP (Cuadros 1-2). En Puebla se ha registrado en Chiautla de Tapia (Cuadro 3).
Pelidnota Mac Leay. P. virescens Burmeister,1844, un ejemplar (junio 2014) sobre vegetación en SP (Cuadros 1-2). En Puebla se ha registrado en Chiautla de Tapia, Cuetzalan, Jolalpan y el Valle de Puebla (Cuadro 3).

\section{Melolonthidae, Rutelinae, Anomalini}

Paranomala Casey. P. forreri Bates, 1888, un ejemplar (junio 2014) sobre vegetación en SP (Cuadros 1-2). En 
Cuadro 3. Número de géneros y especies de escarabaeoideos colectados sistemáticamente en el estado de Puebla (* se refieren sólo especies de Scarabaeidae (necrófilas) y de Trogidae).

\begin{tabular}{|c|c|c|c|c|c|}
\hline Localidad & Vegetación & $\begin{array}{c}\text { Altitud } \\
\text { (m) }\end{array}$ & Géneros & Especies & Autores \\
\hline Chiautla de Tapia & Bosque Tropical Caducifolio bajo & $844-1190$ & 33 & 88 & Cuate-Mozo et al. 2013. \\
\hline Chignahuapan & Bosque de Pino & $2500-2885$ & 16 & 30 & Delgado-Solano et al. 2013. \\
\hline $\begin{array}{l}\text { Citlaltépetl-Ahuatepec- } \\
\text { Tlachichuca }\end{array}$ & Bosque de Pino- Encino & $2600-3780$ & 19 & 31 & Salamanca-Calixto et al. 2013. \\
\hline Cuetzalan & Bosque Tropical Perennifolio & $600-860$ & 34 & 63 & Carrillo-Ruiz \& Morón 2013. \\
\hline Huehuetlán el Grande & $\begin{array}{l}\text { Bosque de Encino, Bosque Tropical } \\
\text { Caducifolio bajo }\end{array}$ & $1300-1400$ & 30 & 54 & Yanes-Gómez \& Morón 2013. \\
\hline Iztaccíhuatl- Teotlalcingo & Bosque de Pino & 2733-3663 & 9 & 23 & Chacón et al. 2013. \\
\hline Jolalapan & Selva Baja Caducifolia & $926-1140$ & 20 & 44 & $\begin{array}{l}\text { Sánchez-Velázquez et al. 2012, } \\
\text { Castañeda-Osorio et al. } 2015 .\end{array}$ \\
\hline Matlalcuéyetl (la Malinche) & $\begin{array}{l}\text { Bosque de Pino, Bosque de Pino- } \\
\text { Encino, Bosque de Oyamel, Bosque de } \\
\text { Oyamel-Pino }\end{array}$ & 2533-3502 & 19 & 36 & García de Jesús et al. 2013. \\
\hline Popocatepétl-Tochimilco & Bosque de Pino- Encino & 2123-2582 & 32 & 70 & Rodríguez et al. 2013. \\
\hline Терехсо* & Bosque tropical deciduo & $1200-1400$ & 12 & 18 & Deloya 1992. \\
\hline Teziutlán & Bosque de Pino- Encino & $1515-1800$ & 29 & 58 & Muñoz-Hernández et al. 2013. \\
\hline Valle de Puebla & Bosque de Pino- Encino & $1700-2350$ & 38 & 98 & Pérez-Torres et al. 2013 \\
\hline Valle de Tehuacán & $\begin{array}{l}\text { Matorral desértico rosetófilo, Matorral } \\
\text { crasicaule, Matorral xerófilo, Bosque } \\
\text { Tropical Caducifolio bajo, Bosque de } \\
\text { Pino-Encino }\end{array}$ & $1200-2120$ & 35 & 72 & $\begin{array}{l}\text { Aragón et al. 2013, } \\
\text { Trujillo-Miranda et al. } 2016 .\end{array}$ \\
\hline Zacatlán & $\begin{array}{l}\text { Bosque de Pino- Encino, Bosque Mesófilo } \\
\text { de Montaña }\end{array}$ & $2040-2500$ & 20 & 40 & Percino-Figueroa et al. 2013. \\
\hline
\end{tabular}

Puebla se ha registrado en la región de Matlalcuéyetl (La Malinche) y el Valle de Puebla (Cuadro 3).

\section{Melolonthidae, Dynastinae, Cyclocephalini}

Cyclocephala Latreille, 1829. C. lunulata Burmeister, 1847, un ejemplar (marzo 2014) sobre el suelo en SP (Cuadros 1-2). En Puebla se ha registrado en Chiautla de Tapia, Cuetzalan, Huehuetlán el Grande, Jolalpan, Popocatépetl-Tochimilco, el Valle de Puebla, Teziutlán y Zacatlán (Cuadro 3).

\section{Dynastinae, Oryctini}

Strategus Hope. S. aloeus Linneo ,1758, tres ejemplares (junio 2014) sobre el suelo en SP (Cuadros 1-2). En Pue- bla se ha registrado en Chiautla de Tapia, Citlaltépetl-Tlachichuca-Ahuatepec, Cuetzalan, Huehuetlán el Grande, Jolalpan, Popocatépetl-Tochimilco, el Valle de Puebla, Tehuacán, Teziutlán y Zacatlán (Cuadro 3).

Xyloryctes Hope. X. telephus, dos ejemplares (junio 2014), sobre el suelo en SP (Cuadros 1-2). En Puebla se ha registrado en Chiautla de Tapia, Iztaccíhuatl-Teotlalcingo, Huehuetlán el Grande, el Valle de Puebla y Tehuacán (Cuadro 3).

\section{Cetoniidae, Cetoniinae, Gymnetini}

Cotinis Burmeister. C. mutabilis Gory \& Percheron, 1833, nueve ejemplares en junio y julio (2014), sobre vegetación en SP (Cuadros 1-2). En Puebla se ha registrado en Chiautla de Tapia, Popocatépetl-Tochimilco, el Valle de Puebla y el Valle de Tehuacán (Cuadro 3). 


\section{Scarabaeidae, Scarabaeinae, Coprini}

Copris Muller. C. lugubris Boheman,1858, 78 ejemplares de noviembre a diciembre (2013) y en enero, febrero, marzo, mayo, junio, julio y noviembre (2014), 77 en SP y uno en SC, todos en boñigas de ganado vacuno (Cuadros 1-2). En Puebla se ha registrado en Chiautla de Tapia (Cuadro 3).

Dichotomius Hope. D. amplicollis Harold, 1869, 29 ejemplares de mayo a julio y octubre (2014), 28 ejemplares en SP y un ejemplar en SC, con necrotrampa (12) y en boñigas de ganado vacuno (17) (Cuadros 1-2). En Puebla se ha reportado en Huehuetlán el Grande, Jolalpan y Tepexco (Cuadro 3); D. colonicus Say, 1835, 11 ejemplares en noviembre (2013), y en febrero, mayo, octubre y noviembre (2014), nueve ejemplares en SP y dos en SC, en boñigas de ganado vacuno (Cuadros 1-2). En Puebla se ha registrado en Chiautla de Tapia, Cuetzalan, Huehuetlán el Grande, Jolalpan, Popocatépetl-Tochimilco, el Valle de Puebla y el Valle de Tehuacán (Cuadro 3). Cabe mencionar que Deloya (1992) reportó a D. centralis (Harold, 1869) en Tepexco, sin embargo, Kohlmann (2003), aclara la diagnosis y validez de $D$. amplicollis y menciona que en México esta especie ha sido erróneamente citada bajo el nombre de $D$. centralis.

Ateuchus Weber. A. rodriguezi de Borre, 1886, 98 ejemplares en noviembre (2013), y de enero a noviembre (2014), 87 ejemplares en SP y 11 ejemplares en SC, con necrotrampa (24) y en boñigas de ganado vacuno (74) (Cuadros 1-2). En Puebla se ha reportado en Chiautla de Tapia, Huehuetlán el Grande, Jolalpan y Tepexco (Cuadro 3). Además, A. halffteri Kohlmann, 1984 fue registrada en Tepexco, por Deloya (1992).

De esta tribu cabe mencionar que Deloya (1992) registró a Canthidium puncticolle Harold, 1868, en Tepexco, especie que no se registró en este estudio.

\section{Scarabaeidae, Scarabaeinae, Phanaeini}

Phanaeus Mac Leay. P. demon Laporte de Castelnau,1840, cinco ejemplares en mayo, junio, julio y septiembre (2014), en boñigas de ganado vacuno en SP (Cuadros 1-2). En Puebla se ha registrado en la región de Chiautla de Tapia y Valle de Puebla (Cuadro 3); P. daphnis Harold, 1863, 28 ejemplares de noviembre a diciembre (2013), de mayo a julio y de septiembre a noviembre (2014), 22 en SP y seis en SC, con necrotrampa (11) y en boñigas de ganado vacuno (17) (Cuadros 1-2). En Puebla se ha registrado en Chiautla de Tapia, Huehuetlán el Grande, Tepexco y el Valle de Puebla (Cuadro 3).

Coprophanaeus Olsoufieff. C. (Coprophanaeus) pluto Harold, 1863, 37 ejemplares de mayo a julio, septiembre y octubre (2014), 19 en SC y 18 en SP, con necrotrampa (Cuadros 1-2). En Puebla se ha reportado en Chiautla de Tapia, Huehuetlán el Grande, Jolalpan, Tepexco, el Valle de Puebla y Zacatlán (Cuadro 3).

\section{Scarabaeidae, Scarabaeinae, Onticellini}

Euoniticellus Janssens. E. intermedius Reiche, 1848, 258 ejemplares en noviembre (2013) y de enero a noviembre (2014), 257 en SP y uno en SC, en boñigas de ganado vacuno (Cuadros 1-2). En Puebla se ha registrado en Chiautla de Tapia, Huehuetlán el Grande, PopocatépetlTochimilco y el Valle de Puebla (Cuadro 3).

Canthon Hoffmannsegg. C. (Glaphyrocanthon) corporaali (Balthasar, 1939), ocho ejemplares en marzo, mayo, junio, julio y noviembre (2014), en boñigas de ganado vacuno en SP (Cuadros 1-2). En Puebla se ha registrado en Chiautla de Tapia, Huehuetlán el Grande, Tepexco, Valle de Puebla (Cuadro 3); C. (Canthon) indigaceus LeConte, 1866, 18 ejemplares recolectados de mayo a julio y septiembre a noviembre (2014), 15 ejemplares en SP y tres en SC, con necrotrampa (11) y en boñigas de ganado vacuno (7) (Cuadros 1-2). En Puebla se ha registrado en Chiautla de Tapia, Cuetzalan, Huehuetlán el Grande, Jolalpan y Valle de Tehuacán (Cuadro 3); C. (Canthon) humectus Say, 1832, 109 ejemplares en noviembre (2013), de abril a noviembre (2014), 90 ejemplares en SP y 19 en SC, con necrotrampa (25) y por recolecta directa (84) (Cuadros 1-2). En Puebla se ha reportado en Chignahuapan, Huehuetlán el Grande, Jolalpan y el Valle de Puebla (Cuadro 3). Además, de este género Deloya (1992) registró en la localidad de Tepexco a C. cyanellus cyanellus Le Conte, 1859, a C. (Glaphyrocanthon) viridis leechi Martínez et al., 1964 y C. humectus incisus Robinson 1948.

Deltochilum Eschscholtz. Representado por D. scabriusculum Bates, 1887, 16 ejemplares de mayo a agosto y noviembre (2014), siete ejemplares en SP y nueve en SC, con necrotrampa (Cuadros 1-2). Esta especie se ha registrado en Puebla (Halffter 2003), sin embargo, no se especifica la localidad. Deloya (1992), además registró a $D$. gibbosum sublaeve Bates, 1887, en Tерехсо. 


\section{Scarabaeidae, Scarabaeinae, Onthophagini}

Onthophagus Latreille. O. mexicanus Bates, 1887, 55 ejemplares en noviembre y diciembre (2013), así como en abril, junio, julio y noviembre (2014), 47 ejemplares en SP y ocho en SC (Cuadros 1-2), con necrotrampa (3) y en boñigas de ganado vacuno (52). En Puebla se ha registrado en Chignahuapan, Huehuetlán el Grande, Matlalcuéyetl (La Malinche), Popocatépetl-Tochimilco, el Valle de Puebla, Valle de Tehuacán y Zacatlán (Cuadro 3). Deloya (1992) registró a O. igualensis Bates, 1887 y a tres especies de Onthophagus no identificadas en Tepexco.

Digitonthophagus Balthasar. D. gazella (Fabricius, 1787), 123 ejemplares en noviembre (2013), así como de abril a noviembre (2014), 122 ejemplares en SP y uno en SC, en boñigas de ganado vacuno (Cuadros 1-2). En Puebla se ha registrado en Chiautla de Tapia, Cuetzalan, Huehuetlán el Grande y Jolalpan (Cuadro 3).

Los Aphodiinae son considerados el grupo dominante en las comunidades de escarabajos del estiércol de las zonas templado-frías de la región Paleártica (Hanski, 1991) y comúnmente, también en las de la región Neártica (Lobo, 2000), pero durante nuestro estudio no logramos capturarlos. Deloya (1992) registró a dos especies en Tepexco, Ataenius platensis (Blanchard, 1843) y Pleurophorus micros (Bates, 1887).

\section{Hybosoridae, Hybosorinae}

Hybosorus Mac Leay. H. illigeri Reiche, 1853, 9 ejemplares en SP (mayo 2014), algunos sobre el suelo cercanos a las boñigas del ganado o bien sobre las boñigas de ganado vacuno (Cuadros 1-2). En Puebla se ha registrado en Chiautla de Tapia, Huehuetlán el Grande y Jolalpan (Cuadro 3).

\section{Trogidae, Troginae}

Omorgus Erichson. O. suberosus (Fabricius,1775), 15 ejemplares en enero, mayo y junio (2014), cuatro ejemplares en SP y 11 en SC (Cuadros 1-2), con necrotrampa. En Puebla se ha registrado en Chiautla de Tapia, Huehuetlán el Grande, Valle de Puebla, Valle de Tehuacán y en Tepexco (Cuadro 3). De este género se ha registrado O. rubricans Robinson, 1946 y O. fuliginosus Robinson, 1942, en Tepexco; así como a Trox spinulosus dentibius Robinson, 1940 (Deloya 1992).

\section{Passalidae, Passalinae}

Ptichopus Kaup. P. angulatus (Percheron, 1835), 22 ejemplares en noviembre y diciembre (2013), así como en marzo, abril, mayo, junio y noviembre (2014), en troncos derribados en SP (Cuadros 1-2). En Puebla se ha registrado en Chiautla de Tapia, Cuetzalan, Huehuetlán el Grande, Popocatépetl-Tochimilco, el Valle de Puebla y el Valle de Tehuacán (Cuadro 3).

Esfuerzo de muestreo. De los 945 ejemplares registrados, 851 se capturaron en el SP y 94 en el SC. Los resultados del Índice de complementariedad mostraron que las especies en los dos sitios de la zona de estudio son distintas (SP-SC= 0.5). Con base en estos resultados se obtuvo la curva de acumulación de especies para toda la zona de estudio, donde el estimador ACE alcanza un 80 $\%$ de esfuerzo de muestreo y predice 35 especies esperadas, por lo que faltan por recolectar 7 especies. Por otro lado, el estimador Chao 1 alcanza un $85 \%$ de esfuerzo de muestreo y predice 33 especies esperadas, por lo que faltan 5 especies por registrar (Fig. 3).

Riqueza, abundancia y diversidad de especies. El SP presentó la mayor riqueza, ya que se capturaron 27 especies de las 28 registradas en ambos sitios. Mientras que en el SC se registraron 15 especies. En el Cuadro 1 se muestran las especies compartidas entre ambos sitios, así como las especies exclusivas a cada uno de ellos.

Los resultados de abundancia relativa mostraron que las especies más abundantes en el SP fueron E. intermedius (30.19\%) con 257 ejemplares, seguida de D. gazella (14.33\%) con 122 ejemplares. Por otra parte en el SC las especies más abundantes fueron $C$. (C.) humectus (20.21\%) y C. (C.) pluto (20.21\%) cada una con 19 ejemplares (Cuadro 1, Fig. 4).

El índice de diversidad de Shannon para el SP fue de 2.31 y para el SC fue de 2.16; al compararse no se encontraron diferencias significativas entre los dos sitios de muestreo $(p=0.111)$.

Diversidad funcional. Los adultos de las especies de escarabajos registradas en la comunidad de Calmeca, se agrupan en ocho gremios alimentarios (Cuadro 4). En ambos sitios la mayoría de las especies registradas pertenecen a los gremios coprófago (SP 37\% y SC 53\%), necrófago (SP 14.8\% y SC 26.6\%) y filófago (SP 18\% y SC $13 \%)$. El SP presentó una mayor riqueza funcional (ocho gremios) que el SC (cuatro gremios); en este último no se registraron especies de los gremios depredador, antófilo, rizófago y detritívoro (Fig. 5). El índice de similitud de Sörensen modificado por Silvestre (2000) mostró que los 


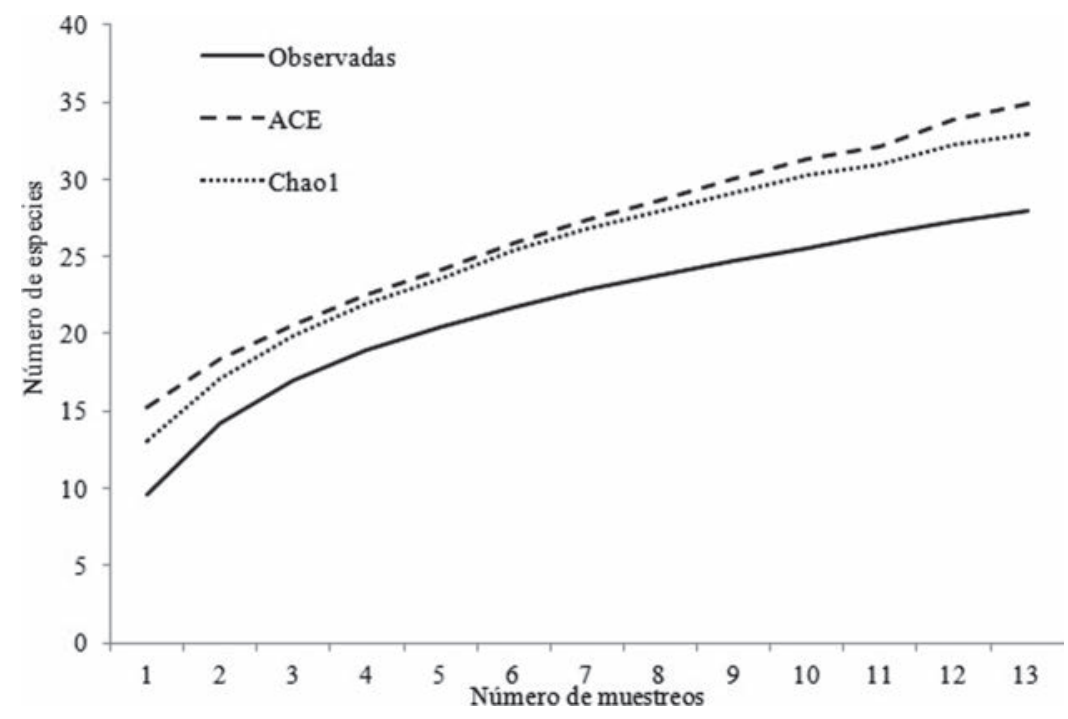

Figura 3. Curva de acumulación de especies de escarabaeoideos registrados en la zona de estudio, de acuerdo a los estimadores Chao 1 y ACE.

dos sitios de muestreo comparten el $40.57 \%$ de los gremios presentes.

\section{DISCUSIÓN}

Esfuerzo de muestreo. La curva de acumulación de especies no alcanzó la asíntota, sin embargo, Verdú y Pineda (2013) consideran que un inventario está razonablemente completo cuando la eficiencia de muestreo es mayor al

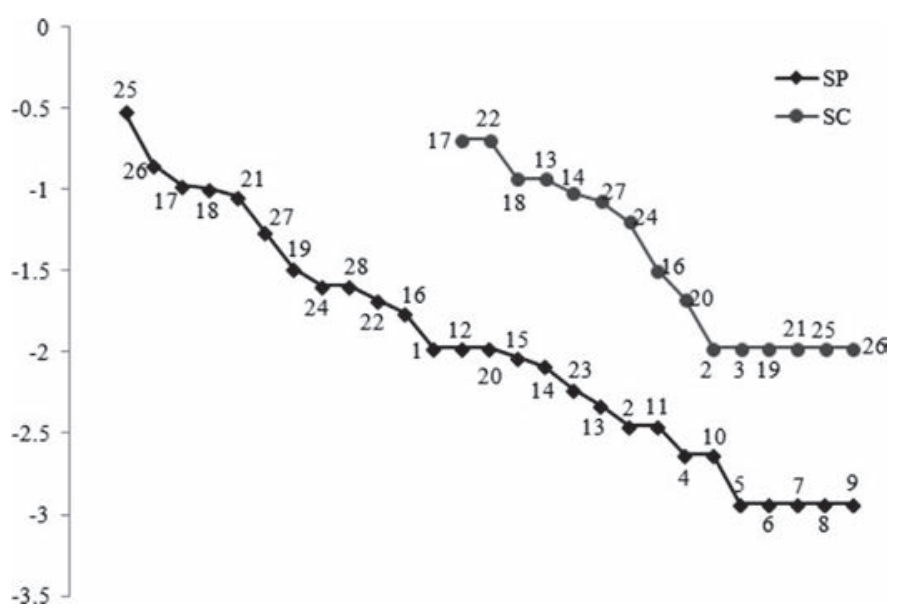

Figura 4. Gráfico rango-abundancia de las especies de escarabaeoideos capturadas en los sitios de estudio, SC= sitio conservado; $\mathrm{SP}=$ sitio modificado (El número corresponde al código de la especie del Cuadro 1).
80\%. En nuestro caso y con el estimador Chao 1 se alcanza un $85 \%$ de eficiencia de muestreo, faltando por recuperar 5 especies. No se empleó trampa de luz, por lo que las especies faltantes pueden ser aquellas que son atraídas a la luz.

Riqueza, abundancia y diversidad de especies. En el SP habitan un mayor número de especies que en el SC, lo cual puede deberse a que este hábitat ofrece diversos recursos, por un lado el excremento del ganado y por otro los cultivos aledaños al mismo. Este tipo de hábitat modificado permite que las larvas de varias especies se alimenten mejor y los adultos se reproduzcan con mayor facilidad (Rivera-Gasperín et al., 2013).

Euoniticellus intermedius y D. gazella son las especies más abundantes en el SP, ambas especies son exóticas (Favila, 2012). Rougon \& Rougon (1982) indican que $E$. intermedius es una especie adaptada a condiciones edáficas y climáticas extremas, mientras que $D$. gazella es una especie de claros con una tasa rápida de reproducción, es oportunista y alta movilidad (Barbero \& López-Guerrero, 1992). Kohlmann (1994) menciona que D. gazella fue capturada por primera vez en México en 1981 Nuevo León y Tamaulipas, y en 1992 se registró por primera vez en Morelos. En el año 2000 se tiene el primer registro de esta especie en montañas húmedas de Puebla (CarrilloRuiz \& Morón, 2003) y entre 2000 y 2003 se encontró en Atencingo, al suroeste de Puebla (Aragón et al., 2012). Con respecto a $E$. intermedius, se tienen los primeros registros confirmados en 1992 para dos localidades de Du- 
Cuadro 4. Gremios alimentarios de las especies colectadas en Calmeca, Tepexco, Puebla, México (* gremio generalizado del género).

\begin{tabular}{|c|c|c|}
\hline Especie & Gremio Alimentario & Autor \\
\hline Diplotaxis consentanea* $^{*}$ & Filófago & Vaurie 1958, Castro-Ramírez et al. 2004. \\
\hline Phyllophaga (Ph.) fulviventris & Filófago & Morón et al. 1996, Morón 1997. \\
\hline Phyllophaga (Ph.) setifera & Filófago & Morón et al. 1996. \\
\hline Calomacraspis concinna & Antófilo & Deloya et al. 1995. \\
\hline Paranomala forreri & Filófago & Pacheco et al. 2006. \\
\hline Cyclocephala lunulata & Antófilo & Ratcliffe \& Morón 1997, Aragón et al. 2010. \\
\hline Strategus aloeus & Rizófago & Ratcliffe 1976, Ratcliffe \& Morón 1997, Bitar \& Morón 2014. \\
\hline Xyloryctes telephus* & Rizófago/Saprófago & Ratcliffe 2009, Bitar \& Morón 2014. \\
\hline Cotinis mutabilis & Antófilo & Morón et al. 1996, Barrales-Alcalá et al. 2012. \\
\hline Ateuchus rodriguezi & Necrófago & Deloya 2003, Trevilla- Rebollar et al. 2010. \\
\hline Phanaeus (P.) demon & Coprófago & Edmonds 1994, Favila 2012. \\
\hline Phanaeus (P.) daphnis & Coprófago & Edmonds 1994, Favila 2012. \\
\hline Coprophanaeus (C.) pluto & Necrófago & Deloya 2003, Mora-Aguilar \& Montes de Oca 2009. \\
\hline Euoniticellus intermedius & Coprófago & Martínez \& Montes de Oca 2013. \\
\hline Canthon (Gl.) corporali & Coprófago & Rivera-Cervantes \& Halffter 1999. \\
\hline Canthon (C.) indigaceus & Coprófago & Halffter 2003. \\
\hline Canthon (C.)humectus & Coprófago & Halffter et al. 2015. \\
\hline
\end{tabular}

rango (Montes de Oca et al., 1994) y otros 25 registros en localidades de Baja California Norte y Sur, Sonora, Chihuahua, Durango, Guanajuato, Tamaulipas, Hidalgo, Michoacán, Jalisco y Veracruz, obtenidos entre 1994 y 1996 (Montes de Oca \& Halffter, 1998). En Puebla se registró por primera ocasión en 1997 en La Cantera, en la Sierra del Tentzo, bajo estiércol de burro (Morón et al., 2000). Al parecer, la biología de estas dos especies, junto con la transformación de los hábitats para uso ganadero, las han hecho exitosas en sitios como el de nuestro estudio (SP), donde el alimento está disponible durante todo el año. Las especies de mayor abundancia en el SC, son dos especies también de Scarabaeidae: C.(C.) humectus, considerada de las más exitosas del Altiplano Mexicano
(Favila, 2012), tiene la capacidad de modificar la tasa de pérdida de calor corporal a medida que aumenta la temperatura (Verdú \& Lobo, 2008), permitiéndole habitar un mayor número de ambientes; $C$. (C.) pluto, es una especie reportada como necro-coprófaga (Edmonds, 2003), por otros autores ha sido reportada como especialista necrófaga (Morón \& Terrón, 1984; Mora-Aguilar \& Montes de Oca, 2009), fue la especie más abundante en el SC, probablemente porque puede aprovechar varios recursos (cadáveres y excremento) disponibles en este hábitat. A pesar de que los sitios estudiados presentan comunidades de escarabaeoideos que difieren en su composición de especies (Cuadro 1), no se encontraron diferencias significativas. Además, de acuerdo al gráfico de rango- 


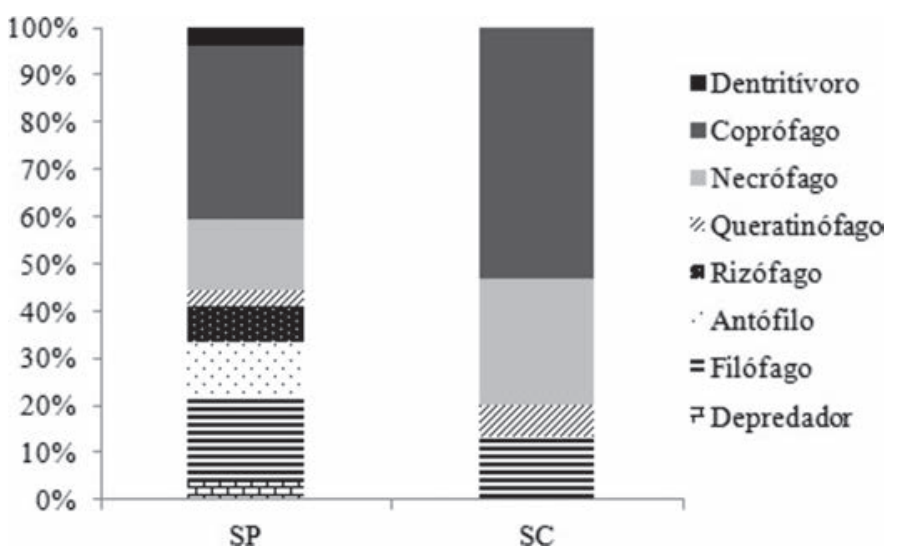

Figura 5. Estructura de los gremios alimentarios en los sitios de estudio, $\mathrm{SC}=$ sitio conservado; $\mathrm{SP}=$ sitio modificado, tras un año de muestreo en Calmeca, Tepexco, Puebla, México.

abundancia (Fig. 4) las especies y sus abundancias son equitativas, en ninguno de los sitios se observan especies con mayor dominancia.

Diversidad funcional. La diversidad funcional permite analizar a las comunidades con base en los diferentes nichos que ocupan las especies en un ecosistema (Petchey \& Gaston, 2006); es una medida de la diversidad de rasgos funcionales presentes en una comunidad con respecto a su presencia y/o abundancia (Córdova-Tapia \& Zambrano, 2015; García-Martínez et al., 2015). La similitud funcional es útil cuando se compara la estructura de la comunidad en el tiempo (o entre sitios), asumiendo que las especies de cada gremio son ecológicamente equivalentes (Martínez, 1996; Silvestre et al., 2003). Esta información proporciona conocimiento valioso acerca de su dinámica y estabilidad frente a perturbaciones (Petchey \& Gaston, 2006). Barragán et al., (2011), en referencia a comunidades de escarabajos copro-necrófagos, indican que la diversidad funcional se puede estimar basándose en la relocalización del alimento, el tamaño del escarabajo, el periodo de actividad diaria y la preferencia del alimento. En este estudio, se determinó emplear la preferencia del alimento de las especies de Scarabaeoidea, considerando los gremios alimentarios como los grupos funcionales (Rivas-Arancibia et al., 2014; CastañedaOsorio et al., 2015; García-Martínez et al., 2015). Así, las especies registradas se agruparon en ocho gremios alimentarios, donde la mayoría de las especies pertenecen al gremio coprófago, seguido del gremio necrófago y en tercer lugar el gremio filófago, habiendo en la comunidad una mayor representación de los gremios que pertenecen al grupo trófico de los degradadores y al de los consu- midores primarios (Morón et al., 2010). Los ocho gremios están representados en el SP, pero solo cuatro de ellos se encuentran representados en el SC. Consideramos probable que las especies faltantes, de acuerdo a los estimadores Chao 1 y ACE, puedan ser integrantes de estos gremios; estos resultados también podrían sugerir que es el ambiente modificado el que proporciona los recursos para que se distribuyan las especies de los diferentes gremios. Probablemente esto pueda ser explicado debido a que el efecto del ganado en los sitios que habitan las comunidades de escarabajos es diferente, ya que depende de las características biogeográficas y ecológicas (Barragán et al., 2011). En nuestro estudio, el SP (sitio de potrero con cultivos aledaños) se ha modificado de tal modo que al parecer ofrece una mayor oportunidad de explotar diferentes recursos por especies con diferentes preferencias alimentarias. Tal es el caso de la especie H. illigeri, reportada como depredadora, cuyos ejemplares fueron capturados sobre o cercanos a las boñigas del estiércol; su presencia puede responder probablemente a la teoría de selección frecuencia-dependiente de la presa, donde la especie depredadora suele tomar la presa más abundante en el hábitat, evitando así un gasto energético al buscar la presa preferida (Begon et al., 1996). La similitud en cuanto a la diversidad funcional de los dos sitios estudiados fue de $40.57 \%$, el cual es un valor bajo e indica que los gremios no están igualmente representados en los dos sitios, se sabe que para el mantenimiento de los procesos ecosistémicos se necesita un número determinado de grupos funcionales, y las especies dentro de esos grupos son parcialmente sustituibles. En nuestro caso, faltan grupos funcionales representados en el SC, por lo que, a pesar de que los indicadores del esfuerzo de muestreo señalan que el inventario es razonablemente completo, consideramos que para tener una mejor aproximación a la dinámica y estructura de las comunidades de Scarabaeoidea es importante aumentar este esfuerzo de muestreo, así como abarcar una mayor área de estudio en la localidad.

AGRADECIMIENTOS. A Rubén Castañeda, por su ayuda en el montaje en seco de los ejemplares capturados.

\section{LITERATURA CITADA}

Aragón, A., Morón, M. A., López-Olguín, J. F. \& Cervantes-Peredo, L. M. (2005). Ciclo de vida y conducta de adultos de cinco especies de Phyllophaga Harris, 1827 (Coleoptera: Melolonthidae; Melolontinae). Acta Zoológica Mexicana (n.s.), 21, 87-99. 
Aragón, A., Lugo-García, G. A., Reyes-Olivas, A., Casillas-Álvarez, P., Villegas-Cota, J. R. \& Morón, M. A. (2010). Huéspedes vegetales de adultos de Coleoptera Scarabaeoidea en el Valle de Carrizo, Sinaloa, México. Southwestern Entomologist, 35, 99-108.

Aragón, A., Morón, M. A., Damián-Huato, M. A., López-Olguín, J. F., Pinson-Rincón, P. \& Pérez-Quintanilla, J. N. (2012). Fauna de Coleoptera Lamellicornia de la zona cañera de Atencingo, Puebla, México. Acta Zoológica Mexicana, 28, 161-171.

Aragón, A., Pérez-Torres, C. B. \& Tapia, A. M. (2013). Capítulo 12. Región del Valle de Tehuacán. Pp. 253-274. In: M. A. Morón, A. Aragón \& H. Carrillo-Ruiz (Eds.). Fauna de escarabajos del estado de Puebla. M.A. Morón, Coatepec, Veracruz, México.

Barbero, E. \& López-Guerrero, Y. (1992). Some considerations in the dispersal power of Digitonthophagus gazella (Fabricius 1787) in the New World (Coleoptera Scarabaeidae Scarabaeinae). Tropical Zoology, 5, 115-120.

Barragán, F., Moreno, C. E., Escobar, F., Halffter, G. \& Navarrete, D. (2011). Negative Impacts of Human Land Use on Dung Beetle Functional Diversity. PloS ONE, 6(3), e17976.

Barrales-Alcalá, D., Carrillo-Angeles, I. G. \& Golubov, J. (2012). Nota sobre Cotinis mutabilis (Coleoptera: Scarabaeidae) alimentándose de frutos de Opuntia robusta (Cactaceae) en Cadereyta, Querétaro, México. Cactáceas y suculentas de México, 57, 86-91.

Begon, M. C., Harper, R. \& Townsend, C. R. (1996). Ecology, individuals, populations and communities. Blackwell Science Ltd. Italy, 876 p.

Bitar, A. \& Morón, M. A. (2014). Revisión y análisis filogenético del género Xyloryctes (Coleoptera: Melolonthidae: Dynastinae: Oryctini). Revista Mexicana de Biodiversidad, 85, 753-796.

Carrillo-Ruiz, H. \& Morón, M. A. (2003). Fauna de Coleoptera Scarabaeoidea de Cuetzalan del Progreso, Puebla, México. Acta Zoológica Mexicana (n.s.), 88, 87-121.

Carrillo-Ruiz, H. \& Morón, M. A. (2013). Capítulo 11. Región de Cuetzalan. Pp. 227-252. In: M. A. Morón, A. Aragón \& H. Carrillo-Ruiz (Eds.). Fauna de escarabajos del estado de Puebla. M. A. Morón, Coatepec, Veracruz, México.

Castañeda-Osorio, R., Carrillo-Ruiz, H., Rivas-Arancibia, S. P. \& Sánchez-Carrillo, M. (2015). Melolonthidae y Cetoniidae (Coleoptera: Scarabaeoidea) del Rancho El Salado, Jolalpan, Puebla, México. Dugesiana, 22, 227-241.

Castro-Ramírez, A. E., Ramírez-Salinas, C. \& Pacheco-Flores, C. (2004). Guía ilustrada sobre "gallina ciega" en la región Altos de Chiapas. El Colegio de la Frontera Sur y Benemérita Universidad Autónoma de Puebla. México, 48 pp.

Chacón, A. L., Aragón, A. \& Morón, M. A. (2013). Capítulo 4. Región del Iztaccihuátl. Teotlalcingo. Pp.83-94. In: M. A. Morón, A. Aragón \& H. Carrillo-Ruiz (Eds.). Fauna de escarabajos del estado de Puebla. M. A. Morón, Coatepec, Veracruz, México.

Colwell, R. K. (2013). EstimateS: Statistical estimation of species richness and shared species from samples. Versión 9. Available at: http://purl.oclc.org/estimates (accessed on July 2016).

Córdova-Tapia, F. \& Zambrano, L. (2015). La diversidad funcional en la ecología de comunidades. Ecosistemas, 24, 78-87.

Cuate-Mozo, V. A., Aragón, A. \& Morón M. A. (2013). Capítulo 14. Región de Chiautla. Pp. 297-322. In: M. A. Morón, A. Aragón \& H. Carrillo-Ruiz (Eds.). Fauna de escarabajos del estado de Puebla. M. A. Morón, Coatepec, Veracruz, México.
Delgado-Solano, J. M., Aragón, A. \& Morón M. A. (2013). Capítulo 8. Región de Chignahuapan. Pp. 167-186. In: M. A. Morón, A. Aragón \& H. Carrillo-Ruiz (Eds.). Fauna de escarabajos del estado de Puebla. M. A. Morón, Coatepec, Veracruz, México.

Deloya, C. (1988). Coleópteros lamelicornios asociados a depósitos de detritos de Atta mexicana (Smith) (Hymenoptera: Formicidae) en el sur del estado de Morelos, México. Folia Entomológica Mexicana, 75, 77-91.

Deloya, C. (1992). Necrophilous Scarabaeoidae and Trogidae beetles of tropical deciduos forest in Tepexco, Puebla, México. Acta Zoológica Mexicana (n.s.), 52, 1-13.

Deloya, C. (2003). Coleoptera Scarabaeidae y Trogidae necrófilos de Valle de Vázquez ("Los Hornos"), Morelos, México. Folia Entomológica Mexicana, 42, 265-272.

Deloya, C., Morón, M. \& Lobo, J. M. (1995). Coleoptera lamellicornia (Macleay, 1819) del sur del estado de Morelos, México. Acta Zoológica Mexicana (n.s.), 65, 1-42.

Diéguez V. \& Gómez R. S. (2004). Aporte al conocimiento de los Trogidae (Coleoptera) de la Argentina. Revista de la Sociedad Entomológica Argentina, 63, 92-95.

Edmonds, W. D. (1994). Revision of Phanaeus Macleay, a new world genus of Scarabaeine dung beetles (Coleoptera: Scarabaeidae, Scarabaeinae). Contributions in Science, Natural History Museum of Los Angeles country, 443, 1-105.

Edmonds, W. D. (2003). Tribu Phanaeini. Pp. 58-65. In: M. A. Morón (Ed.). Atlas de los escarabajos de México. Coleoptera: Lamellicornia. Vol. II Familias Scarabaeidae. Argania Editio, Barcelona.

Favila, M. E. (2012). Historical, biogeographical and ecological factors explain the success of some native dung beetles after the introduction of cattle in Mexico. Pastos, 42, 161-181.

García de Jesús, S., Morón, M. A. \& Aragón, A. (2013). Capítulo 7. Región del Matlalcuéyetl (La Malinche). Pp. 145-166. In: M. A. Morón, A. Aragón \& H. Carrillo-Ruiz (Eds.). Fauna de escarabajos del estado de Puebla. M. A. Morón, Coatepec, Veracruz, México.

García-Martínez, M. A., Martínez-Tlapa, D. L., Pérez-Toledo, G. R., Quiroz-Robledo, L. N., Castaño-Meneses, G., Laborde, J. \& Valenzuela-González, J. E. (2015). Taxonomic, species and functional group diversity of ants in a tropical anthropogenic landscape. Tropical Conservation Science, 8, 1017-1032.

Halffter, G. (2003). Tribu Scarabaeini. Pp. 21-44. In: M. A. Morón (Ed.). Atlas de los escarabajos de México. Coleoptera: Lamellicornia. Vol. II Familia Scarabaeidae. Argania Editio, Barcelona.

Halffter, G., Rivera-Cervantes, L. E. \& Halffter, V. (2015). Diversificación del grupo humectus del género Canthon (Coleoptera: Scarabaeidae: Scarabaeinae) en el occidente de México. Acta Zoológica Mexicana (n.s.), 31, 208-220.

Hammer, Ø., Harper, D. A. T. \& Ryan, P. D. (2001). PAST: Paleontological statistics software package for education and data analysis. Palaeontología Electrónica, 4, 9. Available at: http://nhm2. uio.no/norlex/past/download.html (accessed on July 2016).

Hanski, I. (1991). North temperate dung beetles. Pp. 75-96. In: I. Hanski \& Y. Cambefort (Eds.). Dung beetle ecology. Princeton University Press, Princeton, N J.

Hutcheson, K. (1970). A test for comparing diversities based on the Shannon formula. Journal of theoretical biology, 29, 151-154. 
INAFED-SEGOB. (2010). Enciclopedia de los municipios y delegaciones de México, Puebla. Disponible en: http://www.inafed.gob. mx/work/enciclopedia/EMM21puebla/municipios/21168a.html (Consultado en Julio 2016).

INEGI. (2009), Prontuario de información geográfica municipal de los Estados Unidos Mexicanos, Tepexco, Puebla. Disponible en: http://www3.inegi.org.mx/sistemas/mexicocifras/datos-geograficos/21/21168.pdf (Consultado en Julio 2016).

Jiménez-García, D., Jiménez-García, L. \& Aragón, A. (2013). Capítulo 2. Regiones naturales del estado de Puebla. Pp. 29-54. In: M. A. Morón, A. Aragón \& H. Carrillo-Ruiz (Eds.). Fauna de escarabajos del estado de Puebla. M. A. Morón, Coatepec, Veracruz, México.

Jiménez-Valverde, A. \& Hortal, J. P. (2003). Las curvas de acumulación de especies y la necesidad de evaluar la calidad de inventarios biológicos. Revista Ibérica de Aracnología, 8: 151-161.

Kohlmann, B. (1994). A preliminary study of the invasion and dispersal of Digidonthophagus gazella (Fabricius, 1787) in Mexico (Coleoptera:Scarabaeidae: Scarabaeinae). Acta Zoológica Mexicana (n. s.) 61, 35-42.

Kohlmann, B. (2003). Tribu Coprini. Pp. 45-58. In: M. A. Morón (Ed.). Atlas de los escarabajos de México. Coleoptera: Lamellicornia. Vol. II Familia Scarabaeidae. Argania Editio, Barcelona.

Lobo, J. M. (2000). Species diversity and composition of dung beetle (Coleoptera: Scarabaeoidea) assemblages in North America. The Canadian Entomologist, 132, 307-321.

Lugo-García, G. A., Ortega-Arenas, L. D., González- Hernández, H., Aragón- García, A., Romero- Nápoles, J., Rubio- Cortés, R. \& Morón, M. A. (2011). Melolonthidae nocturnos (Coleoptera) recolectados en la zona agrícola agavera de Jalisco, México. Acta Zoológica Mexicana (n.s.), 27, 341-357.

Magurran, A. E. (2004). Measuring Biological Diversity. Blackwell Publishing company. Oxford, Australia, 256 p.

Martínez, I. \& Montes de Oca, E. (2013). Escarabajos del estiércol. Pp. 40-59. In: M. Cruz \& C. Huerta. Hacia una ganadería sustentable. Estudio de caso: Jilotepec, Veracruz. Instituto de Ecología, A. C. Xalapa, Veracruz, México.

Martínez, N. D. (1996). Defining and measuring functional aspects of biodiversity. Pp. 114-148. In: Gaston, K. J. (Ed.). Biodiversity: a biology of numbers and difference. Blackwell Science, Ltd, London, 148 pp.

Matthews, E. G. 1962. A revision of the genus Copris Muller of the western hemisphere (Coleoptera, Scarabaeidae). Entomologica Americana (NS), 41, 1-139.

Montes de Oca, E. (2001). Escarabajos coprófagos de un escenario ganadero típico de la región de los Tuxtlas, Veracruz, México: importancia del paisaje en la composición de un gremio funcional. Acta Zoológica Mexicana (n.s.), 82, 111-132.

Montes de Oca, E. \& Halffter, G. (1998). Invasion of Mexico by two dung beetles previously introduced into the United States. Studies on Neotropical Fauna \& Environment, 33, 37-45.

Montes de Oca, E., Anduaga, S. \& Rivera, E. (1994). Presence of the exotic dung beetles Euoniticellus intermedius (Reiche) in northern Mexico. Coleopterists Bulletin, 48, 244.

Mora-Aguilar, E. F. \& Montes de Oca, E. (2009). Escarabajos necrófagos (Coleoptera: Scarabaeidae y Trogidae) de la región central baja de Veracruz, México. Acta Zoológica Mexicana (n.s.), 25, 569-588.
Morón, M. A. (1997). Capítulo 6, Melolonthinae. Pp. 205-270. In: M. A. Morón, B. C. Ratcliffe \& C. Deloya (Eds). Atlas de los escarabajos de México. Coleoptera: Lamellicornia. Vol. I. Familia Melolonthidae. CONABIO-SME, México, 280 pp.

Morón, M. A. (2003). Atlas de los escarabajos de México Coleoptera: Lamellicornia Vol. II Familias Scarabaeoidea, Trogidae, Passalidae y Lucanidae. Argania Editio, Barcelona, 227 pp.

Morón, M. A. (2013a). Capítulo 1. Introducción al conocimiento de los escarabajos de Puebla. Pp. 1-28. In: M. A. Morón, A. Aragón \& H. Carrillo-Ruiz (Eds.). Fauna de escarabajos del estado de Puebla. M. A. Morón, Coatepec, Veracruz, México.

Morón, M. A. (2013b). Apéndice II. Clave para identificar las especies de Coleoptera Scarabaeoidea del estado de Puebla. Pp. 415-465. In: M. A. Morón, A. Aragón \& H. Carrillo-Ruiz (Eds.). Fauna de escarabajos del estado de Puebla. M. A. Morón, Coatepec, Veracruz, México.

Morón, M. A. \& Terrón, R. (1984). Distribución altitudinal y estacional de los insectos necrófilos en la Sierra de Hidalgo, México. Acta Zoológica Mexicana (n.s.), 3, 1-47.

Morón, M. A. \& Terrón, R. (1988). Entomología Práctica. Instituto de Ecología, A. C, México. 534 p.

Morón, M. A. \& Deloya, C. 2002. Observaciones sobre el ciclo de vida de Pelidnota (Pelidnota) virescens Burmeister, 1844 (Coleoptera: Melolonthidae; Retelinae). Acta Zoológica Mexicana (n.s.), 85, 109-118.

Morón, M. A., Hernández-Rodríguez, S. \& Ramírez-Campos, A. (1996). El complejo "gallina ciega" (Coleoptera: Melolonthidae) asociado con la caña de azúcar en Nayarit, México. Folia Entomológica Mexicana, 98, 1-44.

Morón, M. A., Aragón, A., Tapia-Rojas, A. M. \& Rojas-García, R. (2000). Coleoptera lamellicornia de la Sierra del Tentzo, Puebla, México. Acta Zoológica Mexicana (n.s.), 79, 77-102.

Morón, M. A., Rodríguez del Bosque, L. A., Aragón, A. \& Ramírez-Salinas, C. (2010). Biología y hábitos de coleópteros Escarabaeoideos. Pp. 65-82. In: L.A. Rodríguez del Bosque \& M. A. Morón (Eds.). Plagas del suelo, Inifap, Colegio de Posgraduados, Universidad Autónoma de Chapingo, Mundi Prensa S.A. de C. V., México, 417 pp.

Muñoz-Hernández, A., Aragón, A. \& Morón, M. A. (2013). Capítulo 10. Región de Teziutlán. Pp. 205-226. In: M. A. Morón, A. Aragón \& H. Carrillo-Ruiz (Eds.). Fauna de escarabajos del estado de Puebla. M. A. Morón, Coatepec, Veracruz, México.

Ocampo, F. (2002). Hybosorids of the United Stated and expanding distribution of the introduced species Hybosorus illigeri (Coleoptera: Scarabaeoidea: Hybosoridae). Annals of the Entomological Society of America, 95, 316-322.

Pacheco, C., Deloya, C. \& Cortés, P. (2006). Phytophagous scarab beetles from the Central Region of Guerrero, Mexico (Coleoptera: Scarabaeidae: Melolonthinae, Rutelinae, Dynastinae, Cetoniinae). Revista Colombiana de Entomología, 32, 191-199.

Percino-Figueroa, S. M. \& Morón, M. A. (2013). Capítulo 9. Región de Zacatlán. Pp. 187-204. In: M. A. Morón, A. Aragón \& H. Carrillo-Ruiz (Eds). Fauna de escarabajos del estado de Puebla. M. A. Morón, Coatepec, Veracruz, México.

Pérez-Torres, C. B., Aragón, A. \& Tapia A. M. (2013). Capítulo 3. Región del Valle de Puebla. Pp. 55-82. In: M. A. Morón, A. Aragón \& H. Carrillo-Ruiz (Eds.). Fauna de escarabajos del estado de Puebla. M. A. Morón, Coatepec, Veracruz, México. 
Petchey, O. L. \& Gaston, K. J. (2006). Functional diversity: back to basics and looking forward. Ecology letters, 9, 741-758.

Quiroz-Rocha, G. A., Navarrete-Heredia J. L. \& Martínez, P. A. (2008). Especies de Scarabaeinae (Coleoptera: Scarabaeidae) y Silphidae (Coleoptera) necrófilas de Bosque de Pino-Encino y Bosque Mesófilo de Montaña en el Municipio de Mascota, Jalisco, México. Dugesiana, 15, 27-37.

Ratcliffe, B. C. (1976). A revision of the genus Strategus (Coleoptera: Scarabaeidae). Bulletin of the University of Nebraska State Museum, 10, 93-204.

Ratcliffe, B. C. (2009). Xyloryctes Hop., 1837 (Coleoptera: Scarabaeidae: Dynastinae: Oryctini) in the United States. Qui es et ubi fuisti et quo vadis? Insecta Mundi, 0100, 1-11.

Ratcliffe, B. C. \& Morón, M. A. (1997). Capítulo 3. Dynastinae. Pp. 53-98. In: M. A. Morón, B. C. Ratcliffe \& C. Deloya. 1997. Atlas de los escarabajos de México. Coleoptera: Lamellicornia. Vol. I. Familia Melolonthidae. CONABIO-SME, México.

Rivas-Arancibia, S. P., Carrillo-Ruiz, H., Bonilla, A. A., Figueroa-Castro, D. M. \& Andrés-Hernández, A. R. (2014). Effect of Disturbance on the Ant Community in a Semiarid Region of Central Mexico. Applied Ecology and Environmental Research, 12, 703-716.

Rivera-Cervantes, L. E. \& Halffter, G. (1999). Monografía de las especies mexicanas de Canthon del subgénero Glaphyrocanthon (Coleoptera: Scarabaeidae: Scarabaeinae). Acta Zoológica Mexicana (n.s.), 77, 23-150.

Rivera-Gasperín, S., Carrillo-Ruiz, H., Morón, M. A. \& YanesGómez, G. (2013). Fauna de Coleoptera Melolonthidae (Scarabaeoidea) en el Rancho Canaletas, Paso del Macho, Veracruz, México. Acta Zoológica Mexicana, 29, 194 -208.

Rodríguez, Y., Aragón, A. \& Morón, M. A. (2013). Capítulo 5. Región del Popocatépetl. Tochimilco. Pp. 95-120. In: M. A. Morón, A. Aragón \& H. Carrillo-Ruiz (Eds.). Fauna de escarabajos del estado de Puebla. M. A. Morón, Coatepec, Veracruz, México

Rojas, P. (1989). Entomofauna asociada a los detritus de Atta mexicana (F. Smith) (Hymenoptera, Formicidae) en una zona árida del centro de México. Acta Zoológica Mexicana (n.s.), 33, 151.

Rougon, D. \& Rougon, C. (1982). Nesting strategies of three species of coprophagous scarabaeinae in the Sahel region of Niger. Pp. 147-150. In: G. Halffter \& W. D. Edmonds (Eds.). The nesting behavior of dung beetles (Scarabaeinae): an ecological and evolutive approach, Instituto de Ecología, México.

Rozas, L., Avila, J. M. \& Sánchez-Piñero, F. (1991). Observación de hábitos depredadores en Hybosorus illigeri Reiche, 1853 (Coleoptera, Scarabaeoidea, Hybosoridae). Boletín de la Asociación Española de Entomología, 15, 111-115.

Salamanca-Calixto, C., Aragón, A. \& Morón M. A. (2013). Capítulo 6. Región del Citlaltépetl. Tlalchichuca y Ahuatepec. Pp.
121-144. In: M. A. Morón, A. Aragón \& H. Carrillo-Ruiz (Eds.). Fauna de escarabajos del estado de Puebla. M. A. Morón, Coatepec, Veracruz, México.

Sánchez-Velázquez, B., Carrillo-Ruiz, H., Morón, M. A. \& RivasArancibia, S. P. (2012). Especies de Scarabaeidae e Hybosoridae (Coleoptera: Scarabaeoidea) que habitan en la comunidad del Rancho El Salado, Jolalpan, Puebla; México. Dugesiana. 18, 207-215.

Silvestre, R. (2000). Estrutura de comunidades de formigas do Cerrado. Tese de Doutorado, Faculdade de Filosofia, Ciências e Letras de Ribeirão Preto da Universodade de São Paulo, São Paulo.

Silvestre, R., Brandao, C. R. F. \& Rosa da Silva, R. (2003). Grupos funcionales de hormigas: el caso de los gremios del Cerrado. Pp. 113-148. In: F. Fernández (Ed.). Introducción a las Hormigas de la región Neotropical. Instituto de Investigación de Recursos Biológicos Alexander von Humboldt. Bogotá, Colombia.

Trevilla-Rebollar, A., Deloya, C. \& Padilla- Ramírez, J. (2010). Coleópteros necrófilos (Scarabaeidae, Silphidae y Trogidae) de Malinalco, Estado de México, México. Neotropical Entomology, 39, 486-495.

Trujillo-Miranda, A. L., Carrillo-Ruiz, H., Rivas-Arancibia, S. P. \& Andrés-Hernández, A. R. (2016). Estructura y composición de la comunidad de escarabajos (Coleoptera: Scarabaeoidea) en el cerro Chacateca, Zapotitlán, Puebla; México. Revista Mexicana de Biodiversidad, 87, 109-122.

Vaurie, P. (1955). A revision of the genus Trox in North America (Coleoptera, Scarabaeidae). Bulletin of the American Museum of Natural History, 106, 1-90.

Vaurie, P. (1958). A revision of the genus Diplotaxis (Coleoptera, Scarabaeidae, Melolonthinae). Bulletin of the American Museum of Natural History, 115, 263-396.

Verdú, J. R \& Lobo, J. M. (2008). Ecophysiology of thermoregulation in endothermic dung beetles: ecological and geographical implications. Pp. 1-28. In: S. Fattorini (Ed.). Insect Ecology and Conservation, Research Signpost, Kerala, India.

Verdú, J. R. \& Pineda-López, R. (2013). Cuaderno de Prácticas. Medición de la biodiversidad: diversidades alfa, beta y gamma. Universidad Autónoma de Querétaro, Universidad de Alicante, Editorial Universitaria, Colección Academia, México.

Villarreal, H., Álvarez, M., Córdoba, S., Escobar, F., Fagua, G., Gast, F., Mendoza, H., Ospina, M. \& Umaña, A. M. (2004). Manual de métodos para el desarrollo de inventarios de biodiversidad. Programa de inventarios de biodiversidad. Instituto de Investigación de Recursos Biológicos Alexander von Humboldt, Bogotá, Colombia, 236 pp.

Yanes-Gómez, G. \& Morón, M.A. (2013). Capítulo 13. Región de Huehuetlán el Grande. Pp. 275-296. In: M. A. Morón, A. Aragón \& H. Carrillo-Ruiz (Eds.). Fauna de escarabajos del estado de Puebla. M. A. Morón, Coatepec, Veracruz, México. 